\title{
Análise dos desfechos materno-fetais em gestantes com ganho ponderal inadequado atendidas no ambulatório de pré-natal da Universidade Brasil, em Fernandópolis, São Paulo, Brasil
}

Analysis of maternal and fetal outcomes in pregnant women with inadequate weight gain attended at the prenatal clinic of the Universidade Brasil, in Fernandópolis, São Paulo, Brazil

Análisis de los resultados maternos y fetales en mujeres embarazadas con aumento de peso inadecuado atendidas en la clínica prenatal de la Universidad de Brasil, en Fernandópolis, São Paulo, Brasil

Bárbara Mayume de SOUSA ${ }^{1}$

Amanda Bergamo BUENO ${ }^{1}$

Amanda Oliva SPAZIANI 1

Matheus Magalhães AZARIAS²

Morisa Martins Leão CARVALHO ${ }^{3}$

${ }^{I}$ Curso de Graduação em Medicina, Universidade Brasil 15600-000 Fernandópolis - SP, Brasil

${ }_{2}^{2}$ Residência em Ginecologia e Obstetrícia do Hospital Ensino Santa Casa de Misericórdia de Fernandópolis 15601-012 - SP, Brasil

${ }^{3}$ Docente de Medicina, Universidade Brasil 15600-000 Fernandópolis - SP, São Paulo, Brasil

\section{Resumo}

Sendo o peso pré-gestacional reconhecido com um dos principais determinantes do ganho ponderal na gestação e o acompanhamento do ganho ponderal nesse período um procedimento acessível e de baixo custo, é de grande utilidade sua análise e controle para possíveis intervenções primárias de prevenção, com promoção à saúde do binômio mãe-feto. Considerando as implicações da obesidade na gestação, esse trabalho objetivou analisar o histórico pré-natal do estado nutricional e possíveis correlações com intercorrências para a saúde materna e a via de parto no Ambulatório de Pré-natal da Universidade Brasil, Fernandópolis, São Paulo, Brasil. Forma avaliados 356 prontuários, considerando as variáveis: índice de massa corpórea na primeira consulta do pré-natal; diagnóstico de diabetes mellitus gestacional; síndromes hipertensivas gestacionais; e a via de parto no período de maio de 2015 a fevereiro de 2017. Dentre a amostra selecionada, 34,2\% das gestantes encontravam-se em estado de sobrepeso e $11 \%$ de obesidade. A prevalência de diabetes mellitus gestacional foi de $4,2 \%$, dentre as quais 33,3\% foram classificadas em sobrepeso e 53,8\% em obesidade. Em relação a síndromes hipertensivas gestacionais, o diagnóstico ocorreu em $10,1 \%$ da amostra, sendo que desta, $47,2 \%$ estavam acima do peso. Já sobre a via de parto, houve prevalência de $46 \%$ de partos operatórios, dentre os quais $56 \%$ ocorreram em gestantes com sobrepeso ou obesidade. Mediante a transversalidade desta temática, já que o excesso de peso na gestação se correlaciona com o aumento de problemas de saúde materna, além do seu impacto econômico para o país, pode-se concluir que atingir ou permanecer em estado nutricional de eutrofia é fundamental para o desenvolvimento saudável do binômio mão-feto e uma boa assistência pré-natal.

Descritores: Gravidez; Peso Corporal; Diabetes Mellitus; Hipertensão; Parto.

\section{Abstract}

Since pre-gestational weight is recognized as one of the main determinants of weight gain in pregnancy and the monitoring of weight gain during this period is an affordable and inexpensive procedure, its analysis and control for possible primary prevention interventions, with promotion, is very useful. to the health of the mother-fetus binomial. Considering the implications of obesity during pregnancy, this study aimed to analyze the prenatal history of nutritional status and possible correlations with complications for maternal health and the mode of delivery at the Prenatal Clinic of the University Brazil, Fernandópolis, São Paulo, Brazil. 356 medical records were evaluated, considering the variables: body mass index at the first prenatal visit; diagnosis of Gestational Diabetes Mellitus; Hypertensive Syndromes in Pregnancy; and the mode of delivery in May 2015. to February 2017. Among the selected sample, $34.2 \%$ of the pregnant women were overweight and $11 \%$ obese. The prevalence of Gestational Diabetes Mellitus was $4.2 \%$, of which $33.3 \%$ were classified as overweight and $53.8 \%$ as obesity. Regarding Hypertensive Syndrome in Pregnancy, the diagnosis occurred in $10.1 \%$ of the sample, $47.2 \%$ of which were overweight. Regarding the way of delivery, there was a prevalence of $46 \%$ of operative deliveries, of which $56 \%$ occurred in overweight or obese pregnant women. Due to the transversality of this theme, since overweight in pregnancy correlates with the increase of maternal health problems, in addition to its economic impact on the country, we can conclude that achieving or remaining in a nutritional state of eutrophy is fundamental for development. healthy hand-fetus binomial and good prenatal care.

Descriptors: Pregnancy; Body Weight; Diabetes Mellitus; Hypertension; Parturition.

\section{Resumen}

Dado que el peso pregestacional es reconocido como uno de los principales determinantes del aumento de peso en el embarazo y el monitoreo del aumento de peso durante este período es un procedimiento económico y económico, su análisis y control para posibles intervenciones de prevención primaria, con promoción, es muy útil. a la salud del binomio madre-feto. Teniendo en cuenta las implicaciones de la obesidad durante el embarazo, este estudio tuvo como objetivo analizar la historia prenatal del estado nutricional y las posibles correlaciones con las complicaciones para la salud materna y el modo de parto en la Clínica Prenatal de la Universidad de Brasil, Fernandópolis, São Paulo, Brasil. . Se evaluaron 356 registros médicos, considerando las siguientes variables: índice de masa corporal en la primera visita prenatal; diagnóstico de diabetes mellitus gestacional; síndromes hipertensivos gestacionales; y la forma de parto desde mayo de 2015 hasta febrero de 2017. Entre la muestra seleccionada, el 34,2\% de las mujeres embarazadas tenían sobrepeso y el $11 \%$ eran obesas. La prevalencia de diabetes mellitus gestacional fue del 4,2\%, de los cuales el 33,3\% se clasificaron con sobrepeso y el 53,8\% en obesidad. Con respecto a los síndromes hipertensivos gestacionales, el diagnóstico se produjo en el 10,1\% de la muestra, de los cuales el 47,2\% tenía sobrepeso. En cuanto a la forma de parto, hubo una prevalencia del $46 \%$ de los partos quirúrgicos, de los cuales el $56 \%$ se produjo en mujeres embarazadas con sobrepeso u obesas. Debido a la transversalidad de este tema, dado que el sobrepeso en el embarazo se correlaciona con el aumento de los problemas de salud materna, además de su impacto económico en el país, se puede concluir que lograr o permanecer en un estado nutricional nutricional es fundamental para Desarrollo saludable del binomio feto-mano y buen cuidado prenatal. Descriptores: Embarazo; Peso Corporal; Diabetes Mellitus; Hipertensión; Parto.

\section{INTRODUÇÃO}

A gestação é um período em que a mulher espera por nove meses um ser que surgiu a partir da junção dos gametas feminino e masculino no momento da fecundação. Os gametas são células haploides, portanto se caracterizam por ter apenas um conjunto de cromossomas, visto serem produzidos por meiose, ao passo que o ovo é diploide. Os órgãos que produzem os gametas chamam-se gônadas. A 
fecundação provoca, no corpo da gestante, diversas alterações fisiológicas, envolvendo transformações de aspectos biológicos e psíquicos que variam individualmente e de acordo com a idade gestacional $^{1}$.

Pontua-se que as alterações fisiológicas advindas no período da gravidez, suaves ou acentuadas, permanecem entre as mais marcantes que o corpo humano pode recepcionar, suscitando receios, equívocos, agonias, mitos ou meramente curiosidade em relação às modificações acontecidas no organismo da mulher. Dessa forma, torna-se imperativo que esses anseios sejam partilhados com um profissional de saúde no momento da assistência pré-natal. Dentre essas modificações fisiológicas da gravidez está o ganho de peso que abrange o crescimento fetal acompanhado por crescimento de tecidos maternos, o que aumenta a demanda energética requerida pela gestante ${ }^{2}$.

Com o aumento do aporte de energia materna é necessário um equilíbrio para satisfazer as necessidades do binômio materno-fetal, para que o bem-estar de ambos não seja comprometido. Devido às evidências de que o estado nutricional inicial préconcepcional e o ganho ponderal na gestação têm implicações diretas na saúde materno-infantil, tornase primordial o controle do peso materno durante o período gravídico ${ }^{3}$.

Nesse sentido, a avaliação nutricional é um dos quesitos essenciais na consulta de pré-natal e compreende cuidados na alimentação voltados à promoção, prevenção, diagnósticos e tratamento de complicações, quando necessário. Essa vigilância nutricional no período gestacional é fundamental para que os profissionais de saúde consigam identificar precocemente fatores de risco e agir sobre eles. Desvios no estado materno pré-gestacional e no ganho ponderal durante a gestação estão relacionados a complicações como síndromes hipertensivas gestacionais, diabetes mellitus gestacional e desenvolvimento inadequado do feto ${ }^{4}$.

De acordo Organização Mundial da Saúde, acompanhar o ganho ponderal no período gestacional é um procedimento acessível por ser de baixo custo e de grande utilidade para introdução de medidas nutricionais adequadas para reduzir riscos maternos e fetais, proporcionando um ganho de peso adequado e prevenindo o ganho excessivo ou diagnosticando o ganho ponderal insuficiente 5 .

A Academia Nacional de Medicina dos Estados Unidos (em inglês: National Academy of Medicine) - conhecida como Institute of Medicine (IOM) -, no documento intitulado Nutrition During Pregnancy: Part I Weight Gain: Part II Nutrient Supplements (1990), reconhece o peso prégestacional como um dos principais determinantes do ganho ponderal na gestação e recomenda que o ganho de peso adequado seja analisado em função do estado nutricional inicial da gestante, sendo este definido de acordo com as categorias de índice de massa corpórea (IMC) pré-concepcional ${ }^{6}$.

O IMC é um importante mecanismo de avaliação do estado nutricional de adultos, calculado pela divisão do valor da massa corporal em quilogramas pelo quadrado da estatura em metros (IMC $=\mathrm{kg} / \mathrm{m} 2)$. A Organização Mundial da Saúde, o IOM, a Society of Obstetricians and Gynaecologists of Canada e o Ministério da Saúde do Brasil recomendam diferentes ganhos ponderais de acordo com o estado nutricional pré-gestacional, que é avaliado pelo IMC. Mulheres que apresentam baixo peso devem ter um ganho ponderal de $12,5 \mathrm{~kg}$ a 18,0 $\mathrm{kg}$; gestantes de peso adequado de $11,5 \mathrm{~kg}$ a $16,0 \mathrm{~kg}$; mulheres com sobrepeso de $7,0 \mathrm{~kg}$ a $11,0 \mathrm{~kg}$; e gestantes obesas devem apresentar ganho menor ou igual a $7,0 \mathrm{~kg}^{5}$.

O ganho ponderal inadequado na gestação possui dois extremos, o baixo peso materno que é considerado como fator de risco para as complicações e resultados adversos ocorridos na gestação, especialmente o baixo peso ao nascer. E a obesidade, distúrbio metabólico caracterizado pelo excesso de tecido adiposo, que durante a gravidez relaciona-se com o aumento das taxas de partos operatórios, elevação do risco de complicações perinatais, como maior prevalência de fetos macrossômicos, desproporção céfalo-pélvica, trauma, asfixia e morte perinatal. Além de maior associação com diabetes mellitus gestacional e síndromes hipertensivas gestacionais ${ }^{7}$.

Considerando as implicações do ganho ponderal gestacional e o peso pré-gravídico nos desfechos maternos, no parto e no desenvolvimento fetal, justifica-se aqui a realização de análise nos desfechos insatisfatórios alcançados na gestação, no parto e no desenvolvimento fetal mediante ao ganho ponderal inadequado no período gestacional, objetivando reduzir as possíveis complicações do binômio mãe e feto.

Diante do exposto, este estudo objetivou analisar o histórico pré-natal nutricional e suas consequências para o concepto e para as gestantes acompanhadas no ambulatório de pré-natal da Universidade Brasil, em Fernandópolis, São Paulo, Brasil, no período de maio de 2015 a fevereiro de 2017. Considerando ainda avaliar o estado nutricional das gestantes acompanhadas no ambulatório de prénatal através do IMC da primeira consulta do prénatal; analisar os desfechos maternos com diabetes mellitus gestacional e síndromes hipertensivas gestacionais; analisar as interferências nas variáveis intraparto - via de parto e intercorrências; e analisar complicações no desenvolvimento.

MATERIAL E MÉTODO

Foi realizado um estudo descritivo e 
transversal por meio da pesquisa documental nos prontuários de gestantes atendidas no Ambulatório de Pré-Natal da Universidade Brasil de Fernandópolis, SP, associada a investigação bibliográfica sobre o tema, mediante a importância do assunto abordado.

Foram avaliados 356 prontuários, selecionando amostra composta por gestantes em estado nutricional de sobrepeso ou obesidade de acordo com o com IMC constado na primeira consulta do prénatal que receberam assistência adequada, incluindo o mínimo de seis consultas, de acordo com o Ministério da Saúde, e os exames complementares necessários e de rotina no serviço. O período de coleta foi de maio de 2015 a fevereiro de 2017, de acordo com o material disponível para análise. Foram excluídas as gestantes que abandonaram o acompanhamento pré-natal e aquelas sem registro dos dados necessários à análise nos prontuários.

A análise quantitativa foi realizada pelas recomendações do IOM, as quais implicam que o que o ganho de peso durante a gestação seja diferenciado segundo o estado nutricional pré-gestacional 1.8

Também foi considerada a curva de Atalah, desenvolvida por E. S. Atalah, C. L. Castillo, R. S. Castro e A. P. Aldea, em Propuesta de un Nuevo Estándar de Evaluación Nutricional en Embarazadas (1997), que classifica o estado nutricional da gestante se baseando no peso, altura e idade gestacional; sendo para estado nutricional de baixo peso com IMC $<18,5 \mathrm{~kg} / \mathrm{m} 2$; adequado com IMC entre 18,5-24,9 $\mathrm{kg} / \mathrm{m} 2$; sobrepeso com IMC $>24,9 \mathrm{~kg} / \mathrm{m} 2$; e obesa com IMC $\geq 30 \mathrm{~kg} / \mathrm{m}^{9}$.

Foram considerados desfechos gestacionais as complicações maternas resultantes do processo gestacional, incluindo diabetes, hipertensão e tipo de parto. Adotando como diabetes gestacional a presença de glicemia de jejum $>126 \mathrm{mg} \%$ ou alteração da curva glicêmica ${ }^{11}$. A hipertensão foi definida como pressão sistólica igual ou superior a $140 \mathrm{mmHg}$ e/ou diastólica igual ou superior a 90 $\mathrm{mmHg}^{11}$.

\section{RESULTADOS E DISCUSSÃO}

No Ambulatório de Pré-Natal da Universidade Brasil, Fernandópolis, SP, foram avaliados 356 prontuários, dos quais foram encontradas: 7 gestantes $(2 \%)$ em estado nutricional de baixo peso (IMC $<18,5 \mathrm{~kg} / \mathrm{m} 2$ ), 188 gestantes $(52,8 \%)$ em estado nutricional adequado (IMC entre $18,5-24,9 \mathrm{~kg} / \mathrm{m} 2) ; 122$ gestantes $(34,2 \%)$ com sobrepeso (IMC > 24,9 kg/m2); e 39 gestantes $(11 \%)$ obesas (IMC $\geq 30 \mathrm{~kg} / \mathrm{m} 2$ ), de acordo com os dados anotados no primeiro encontro do pré-natal.

Dentre as gestantes com IMC acima do adequado, $75,8 \%$ encontravam-se em estado nutricional de sobrepeso e $24,2 \%$ eram obesas. A prevalência de diabetes mellitus gestacional nas gestantes analisadas foi de 4,2\% (15), dentre as quais
$13,3 \%$ (2) eram classificadas em estado nutricional adequado, $33,3 \%$ (5) com sobrepeso e 53,3\% (8) com obesidade.

A hiperglicemia durante a gravidez pode variar dependendo dos critérios diagnósticos utilizados e da população estudada. De acordo com a Organização Mundial de Saúde, a prevalência de diabetes mellitus gestacional de mulheres acima de 20 anos atendidas no Sistema Único de Saúde é de $7,6 \%{ }^{12}$.

Um estudo realizado em Brasília, DF, demonstrou que $6,6 \%$ das gestantes atendidas nos centros de saúde básica apresentaram diagnóstico de diabetes mellitus gestacional ${ }^{13}$. Em outro estudo, onde os autores analisam os mais recentes avanços no tratamento do diabetes gestacional, a incidência foi variável, sendo de $3 \%$ a $8 \%$ das gestantes analisadas ${ }^{14}$. Assim, sendo a prevalência de diabetes mellitus gestacional no presente estudo de 4,2\%, conclui-se que os dados corroboram o que diz a literatura atual.

Quanto à prevalência de síndromes hipertensivas gestacionais, neste estudo foi de 36 gestantes $(10,1 \%)$, sendo que 17 gestantes $(47,2 \%)$ com IMC $>25 \mathrm{~kg} / \mathrm{m} 2$ e 19 gestantes $(52,8 \%)$ com $\mathrm{IMC}<25 \mathrm{~kg} / \mathrm{m} 2$. Um estudo realizado na Universidade Federal de Goiás apresentou prevalência de $14,5 \%$ de síndromes hipertensivas nas gestantes atendidas pelo Hospital das Clínicas de Goiânia $^{15}$.

Em Campina Grande, PB, a hipertensão arterial foi observada em $8,5 \%$ da amostra em um estudo que avaliou as gestantes do Programa Saúde da Família ${ }^{16}$. Já no Rio de Janeiro, RJ, um estudo demonstrou $9,6 \%$ de prevalência de Hipertensão Arterial em gestantes, sendo que o sobrepeso/obesidade estava presente em 39\% dessas pacientes ${ }^{17}$.

Neste estudo, a prevalência de síndromes hipertensivas gestacionais nas gestantes avaliadas foi de $10,1 \%$, das quais $47,2 \%$ encontravam-se em estado nutricional de sobrepeso/obesidade. A maior variação de dados, comparando com o estudo realizado no Hospital das Clínicas de Goiânia, pode ser atribuída aos diferentes níveis de assistência à saúde explorados (nível terciário x nível primário). Ademais, nota-se que a síndromes hipertensivas gestacionais tem prevalências niveladas em diferentes locais do Brasil, ratificando a magnitude problemática dessa condição para a promoção da saúde da gestante no país.

Em relação à distribuição dos partos operatórios de acordo com o peso das gestantes analisadas neste estudo, houve prevalência de $46 \%$ de partos operatórios (164), dentre os quais $56 \%$ foram em gestantes com sobrepeso/obesidade e $44 \%$ peso adequado. No estudo realizado em Campinas, SP, com gestantes obesas demonstrou prevalência de 
57,7\% de cesáreas, o que está de acordo com o observado no estudo aqui disposto, ratificando o elevado número de cesáreas no Brasil ${ }^{18}$.

De acordo com o Sistema de Informações de Nascidos Vivos, que engloba informações epidemiológicas referentes aos nascimentos informados em todo território nacional, a taxa brasileira de parto operatório aumentou de 39\% (em 1994) para $44 \%$ (em 2005) $^{19}$. Na presente análise, a via de parto apresentou correlação com o IMC da primeira consulta do pré-natal, sendo a cesariana mais prevalente quanto maior o índice.

Considerando a elevada e progressiva prevalência do sobrepeso e da obesidade na população brasileira, sendo que aproximadamente $50 \%$ destas mulheres em idade fértil encontram-se acima do peso, é consoante que a gestação seja tratada com toda a atenção pelas autoridades de saúde no $\mathrm{Brasil}^{20}$. Mediante a transversalidade desta temática, já que o excesso de peso na gestação se correlaciona com o aumento de problemas de saúde materna, além do impacto econômico do aumento da realização de exames e procedimentos e da utilização de medicamentos, e sendo uma questão de considerável vulnerabilidade no âmbito da saúde, a intensificação das ações primárias de prevenção poderia colaborar com a diminuição da prevalência desse problema ${ }^{21}$.

\section{CONCLUSÃO}

Tendo em vista as intercorrências correlacionadas entre gestação e o estado nutricional materno, pode-se concluir que atingir ou permanecer em estado nutricional de eutrofia é fundamental para o desenvolvimento saudável do binômio mão-feto e uma boa assistência pré-natal.

\section{REFERÊNCIAS}

1. Silva LS, Pessoa FB, Pessoa DTC. Análise das mudanças fisiológicas durante a gestação: desvendando mitos. Rev FMB. 2015;8(1):1-16.

2. Santos AL, Radovanovic CAT, Marcon SS. Assistência pré-natal: satisfação e expectativas. Rev Rene. 2010;11(Esp.):61-71.

3. Sawada M, Masuyama H, Hayata K, Kamada Y, Nakamura K, Hiramatsu Y. Pregnancy complications and glucose intolerance in women with polycystic ovary syndrome. Endocr J. 2015;62(11):1.017-2.

4. Laporte-Pinfildi ASC, Zangirolani LTO, Spina N, Martins PA, Medeiros MAT. Atenção nutricional no pré-natal e no puerpério: percepção dos gestores da Atenção Básica à Saúde. Rev Nutr. 2016;29(1):10923.

5. Gonçalves CV, Mendoza-Sassi RA, Cesar JA, Castro NB, Bortolomedi AP. Índice de massa corporal e ganho de peso gestacional como fatores preditores de complicações e do desfecho da gravidez. Rev Bras Ginecol Obstet. 2012;34(7):304-9.
6. Santos JGC, Silva JMC, Passos AMPR, Monteiro BKSM, Maia MM, Silva RA et al. Peso materno em gestantes de baixo risco na atenção pré-natal. Int J Nutrology. 2017;10(2):5-15.

7. Lima EM. Assistência nutricional no pré-natal: avaliação do processo nas Unidades de Saúde da família no município de Vitória de Santo Antão, PE. Vitória de Santo Antão: Universidade Federal de Pernambuco; 2015.

8. Asbee SM, Jenkins TR, Butler JR, White J, Elliot M, Rutledge A. Preventing excessive weight gain during pregnancy through dietary and lifestyle counseling. Obstet Gynecol. 2009;113(2 Pt 1):30512.

9. Godoy AC. Ganho de peso gestacional recomendações e adequação entre mulheres brasileiras [dissertação] . Campinas: Universidade Estadual de Campinas, UNICAMP; 2015.

10. World Health Organization. Obesity: Preventing and managing the global epidemic: report of a WHO Consultation. Geneva: WHO Consultation on Obesity; 2000.

11. National High Blood Pressure Education Program Working Group on High Blood Pressure in Pregnancy. Report of the National High Blood Pressure Education Program Working Group on High Blood Pressure in Pregnancy. Am J Obstet Gynecol. 2000;183(1):S1-22.

12. Santos EMF, Amorim LP, Costa OLN, Oliveira N, Guimarães AC. Perfil de risco gestacional e metabólico no serviço de pré-natal de maternidade pública do Nordeste do Brasil. Rev Bras Ginecol Obstet. 2012;34(3):102-6.

13. Valladares CG, Konka SB. Prevalência de diabetes mellitus gestacional em gestantes de um centro de saúde de Brasília - DF. Comun Ciênc Saúde. 2008;19(1):11-7.

14. Maganha CA, Vanni DG, Bernadini MA, Zugaib M. Tratamento do diabetes mellito gestacional. Rev Assoc Med Bras. 2003;49(3):330-34.

15. Assis TR, Viana FP, Rassi S. Estudo dos principais fatores de risco maternos nas síndromes hipertensivas da gestação. Arq Bras Cardiol. 2008;91(1):11-7.

16. Melo ASO, Assunção PL, Gondim SSR, Carvalho DF, Amorim MMR, Benicio MHD'A et al. Estado nutricional materno, ganho de peso gestacional e peso ao nascer. Rev Bras Epidemiol. 2007;10(2):249-57.

17. Vettore MV, Dias M, Domingues RMSM, Vettore MV, Leal MC. Cuidados pré-natais e avaliação do manejo da hipertensão arterial em gestantes do SUS no Município do Rio de Janeiro, Brasil. Cad Saúde Pública. 2011;27(5):1.021-34.

18. Job HGC, Passini R Jr; Pereira BG. Obesidade e gravidez: avaliação de um programa assistencial. Rev Ciênc Méd. 2005;14(6):503-14.

19. Brasil. Ministério da Saúde. Pesquisa Nacional de 
Demografia e Saúde da Criança e da Mulher PNDS 2006: dimensões do processo reprodutivo e da saúde da criança. Brasília, DF: Ministério da Saúde; 2009 [citado 2019 Set 26]. Disponível em: http://bvsms.saude.gov.br/bvs/publicacoes/pnds_cria nca_mulher.pdf

20. Carneiro JRI, Braga FO, Cabizuca CA, Abi-Abib RC, Cobas RA, Gomes MB. Gestação e obesidade: um problema emergente. Rev HUPE. 2014;13(3):1724.

21. Chu SY, Bachman DJ, Callaghan WM, Whitlock EP, Dietz PM, Berg CJ et al. Association between obesity during pregnancy and increased use of health care. N Engl J Med. 2008;358(14):1444-53.
Os autores declaram não haver conflitos de interesse.

\section{AUTOR PARA CORRESPONDÊNCIA}

Amanda Oliva Spaziani

Faculdade de Medicina, Campus Fernandópolis

Universidade Brasil

E-mail: spazianimedicina@gmail.com
Submetido em 29/09/2019

Aceito em 20/04/2020 